\title{
Abbreviated Care-Process Quality Indicator Sets Linked with Survival and Functional Status Benefit in Older Adults Under Ambulatory Care
}

\author{
Lillian Min, MD, MSHS,,$^{a, b}$ David Reuben, MD, ${ }^{c}$ Arun Karlamangla, MD, PhD ${ }^{c}$ Arash Naeim, \\ MD, PhD, ${ }^{d}$ Katherine Prenovost, PhD, ${ }^{e}$ Pearl Lee, MD, MS, ${ }^{a, b}$ and Neil Wenger, MD ${ }^{d, f}$
}

OBJECTIVES: To identify subsets of ambulatory care (outpatient only) quality indicators (QIs) associated with better survival and physical function outcomes.

DESIGN: Observational cohort study.

SETTING: Pooled data from the Assessing Care of Vulnerable Elders (ACOVE)-1 study, which measured quality of care using 140 care-process QIs, and the subsequent ACOVE-2 study, which reduced the QIs to 69 ambulatory care QIs.

PARTICIPANTS: Older adults receiving ambulatory care $(\mathrm{N}=1,015)$.

MEASUREMENTS: To prioritize and reduce the QIs into subsets, an expert panel rated each of 69 ambulatory care QIs for the strength of the link between process and benefit, defined as direct trial evidence on older adults or high expectation of benefit if a trial were conducted in older adults. This resulted in three reduced QI sets, reflecting their intended benefit: 17 QIs for survival (ACOVE Quality for Survival (AQS)-17), five QIs to preserve function (AQF-5), and 16 QIs to improve quality-of-life related to physical health and symptoms (AQQ-16). Whether AQS17 would predict 3-year survival was first tested in 1,015 pooled ACOVE-1 and ACOVE-2 participants. Second, whether AQF-5 $(\mathrm{n}=74)$ and AQQ-16 ( $\mathrm{n}=359)$ would predict change in the Physical Component Summary (PCS) score of the Medical Outcomes Study 12-item Short-Form

From the a Division of Geriatric and Palliative Medicine, Department of Medicine, School of Medicine, University of Michigan, ${ }^{b}$ Geriatrics Research, Education and Clinical Care Center, Ann Arbor Veterans Affairs Healthcare System, Ann Arbor, Michigan; 'Division of Geriatrics, Department of Medicine, David Geffen School of Medicine, University of California at Los Angeles, ${ }^{\mathrm{d}}$ Division of General Internal Medicine and Health Services Research, Department of Medicine, David Geffen School of Medicine, University of California at Los Angeles, Los Angeles,

${ }^{\mathrm{e}}$ University of Michigan, Ann Arbor, Michigan; and ${ }^{\mathrm{f}}$ RAND Health, Santa Monica, California.

Address correspondence to Lillian Min, Division of Geriatric and Palliative Medicine, Department of Medicine, School of Medicine, University of Michigan, 300 N. Ingalls, Ann Arbor, MI 48109. E-mail: lmin@med.umich.edu

DOI: $10.1111 /$ jgs.12943
Survey at 1 year was tested in the ACOVE-2 cohort. Control variables were age, function-based vulnerability, and comorbidity.

RESULTS: Each 20-percentage-point increment in AQS17 was associated with survival (hazard ratio (HR) $=0.83, P=.01)$ up to 500 days but not thereafter. AQF5, but not AQQ-16, predicted 1-year improvement in PCS score (1.13-points per 20\%-point increment in AQF-5, $P=.02)$.

CONCLUSION: Subsets of care processes can be linked with outcomes important to older adults. The AQS-17 and AQF-5 are potential tools for improving ambulatory care of older adults. J Am Geriatr Soc 62:1442-1450, 2014.

Key words: quality indicators; geriatric; mortality; physical function

B etter comprehensive quality of medical care for older adults with multiple chronic conditions has been linked to better outcomes. ${ }^{1-3}$ In the Assessing the Care of Vulnerable Elders Study (ACOVE-1), composite scores based on 140 care-process quality indicators (QIs) spanning 22 conditions and ambulatory and hospital care ${ }^{4}$ were associated with better 3 -year survival. ${ }^{1}$ Similarly, better quality of care (composite of 120 QIs) of middle-aged chronically ill adults has been linked to better healthrelated quality of life (HRQOL). ${ }^{2}$ In older adults eligible for nursing home placement, high-quality care has been linked to better physical function and survival. ${ }^{3}$

Nevertheless, there is increasing concern that application of multiple clinical guidelines and QIs overburdens older adults with multiple chronic conditions. ${ }^{5,6}$ In ACOVE-1, individuals with three or more conditions qualified for more than $30 \mathrm{QIs}^{7}$ - with nearly all QIs representing more (not less) recommended care. Furthermore, the need to reduce the ACOVE QIs to a core set of highpriority ambulatory care QIs (e.g., <30) that could be 
implemented feasibly as a starting point for quality improvement for populations of individuals was identified.

One proposed approach is to prioritize care processes with the greatest clinical benefit, ${ }^{8}$ but ambulatory care QIs vary greatly with respect to intended clinical benefits. Although some QIs are aimed at prolonging survival, others (e.g., counseling about advanced directives), are unlikely to be associated with longer survival. Therefore, survival, HRQOL, and functional status were used to identify smaller subsets of QIs that would be associated with these outcomes, guiding future efforts to improve care. Addressing older adults' varying preferences for healthcare benefits would enhance the quality improvement toolbox.

\section{METHODS}

\section{Overview}

This was a secondary analysis of data pooled from two longitudinal ACOVE studies, approved by appropriate institutional review boards.

\section{Samples}

ACOVE- $1^{4}$ and ACOVE- $2^{9}$ have been previously described. ACOVE-1 tested the feasibility of measuring 13 months of ambulatory and acute quality of care (QOC) using medical records from 372 participants aged 65 and older. Using the Vulnerable Elders Survey-13 (VES-13), a 13-item questionnaire based on functional status, ${ }^{10}$ ACOVE-1 screened two large managed care organizations for the top one-third of individuals most vulnerable to death and functional decline. ACOVE-1 found that older adults received poorer care for geriatric conditions (falls, dementia, urinary incontinence) than general medical conditions, ${ }^{4}$ so ACOVE- 2 was conducted to improve geriatric care in primary care practices.

ACOVE-2 measured ambulatory QOC using medical records from 644 individuals aged 75 and older in two large multispecialty practices. In contrast to ACOVE-1, participants were prospectively screened for three geriatric conditions (urinary incontinence, dementia, falls) rather than using the VES-13. Other differences are summarized in Table 1. ACOVE-2 included a controlled practiceimprovement intervention that targeted the care process of primary care clinicians and administrative staff rather than participants themselves. ${ }^{11}$ The intervention improved the QOC of falls and urinary incontinence but not dementia ${ }^{9}$ and did not result in unintended decrement in QOC for nonintervention conditions. ${ }^{12}$

By combining ACOVE-1 and ACOVE-2 data (1,015 total participants, Figure 1) the effects of QOC and comorbidity effects on survival were tested. ACOVE-2 data only (Figure $1, \mathrm{n}=644$ ) were used to test QOC measures aimed at preserving physical function and HRQOL.

\section{Quality Measurement}

QI development for vulnerable elderly adults has been previously described. ${ }^{10}$ ACOVE QIs measure whether clinical care processes (e.g., prescribing medications or ordering tests), rather than outcomes of health care (e.g., glycemic control, mortality), were performed. ${ }^{4}$ In ACOVE-2, the 140 QIs were streamlined to 69 ambulatory care QIs ${ }^{4}$ (Figure 1) concerning 12 areas of outpatient preventive and chronic disease management (Table 1).

In both ACOVE studies, the QIs were applied to measure QOC for 13 months of documented medical care. Of the 69 QIs, 12 were aimed at primary prevention (e.g., vaccinations) or continuity of care (e.g., advanced directives) and therefore were measured in more participants. The remainder were measured based on eligibility criteria (triggered) based on a participant's medical diagnoses. Therefore, a participant with multimorbidity triggered more QIs than a participant with no chronic conditions. If a participant refused or could not tolerate recommended care, the QI was still considered to have been passed. A subset of QIs was excluded from scoring (not triggered) based on appropriateness criteria for participants with advanced dementia or less than 6 months life expectancy. ${ }^{13}$ A participant-level quality score was calculated for each participant as number of QIs passed divided by number of QIs triggered.

\section{Expert Panel Voting for Intended Clinical Benefit}

Literature review and expert panel voting was previously used to establish the validity and appropriateness ${ }^{14-17}$ of ACOVE QIs in older adults and to categorize QIs according to condition (e.g., diabetes mellitus, dementia) and care process (e.g., ordering tests, counseling).4,18,19 For this project, a panel of five physicians (geriatrics, internal medicine) with expertise in QI development and health outcomes of older adults was convened. The panel reconsidered the evidence for each QI and three benefits: survival, preservation of functional status, and HRQOL or physical symptoms. Using literature that previously supported each ACOVE ambulatory care QI, the panel voted on whether each QI was known to have direct link to the benefit in prior clinical trials of older adults or would be strongly expected to be linked to the benefit if a trial of vulnerable older adults was conducted. A QI with neither rating indicated that the care process was not linked to that benefit. A link was said to exist between a QI and a benefit if at least four of the five raters rated the QI as being known or strongly expected to be linked with the benefit. A QI could be linked to none, one, or more than one benefit. The expert panel linked 18 QIs to survival, five to preserving functional status, and 16 to improving HRQOL or symptoms (Table 2 and Figure 1), considering a less than 5-year time frame. Full text of the QIs linked to benefit are included in Appendix S1. The QIs linked with any benefit were considered for inclusion as part of a new benefit-oriented composite QOC score.

\section{Predictor Measures}

The primary predictors of interest were participant-level QOC scores, calculated as the number of QIs passed divided by the number of QIs for which that participant was eligible. Of 18 QIs rated as linked to survival (Table 2), 17 were measured in ACOVE-1 and ACOVE-2 and used to calculate the ACOVE Quality-for-Survival 
Table 1. Comparisons of the Assessing Care of Vulnerable Elders (ACOVE)-1 and 2 Data Sets Before and After Pooling

Before Pooling

\begin{tabular}{|c|c|c|c|}
\hline \multirow[b]{2}{*}{ Element } & \multicolumn{2}{|c|}{ Detore roming } & \multirow{2}{*}{$\begin{array}{l}\text { Pooled Sample for Survival } \\
\text { Outcomes, } N=1,015\end{array}$} \\
\hline & ACOVE-1, N = 372 & ACOVE-2, $N=644$ & \\
\hline Setting & $\begin{array}{l}\text { Community-dwelling vulnerable } \\
\text { elderly adults screened from } 2 \\
\text { managed care organizations }\end{array}$ & $\begin{array}{l}\text { Large group practices ( } 2 \text { groups } \\
\text { at } 7 \text { sites) screened for } \\
\text { symptoms of } 3 \text { geriatric } \\
\text { syndromes (falls, dementia, UI) }\end{array}$ & \\
\hline Age & $\geq 65($ mean $81 \pm 6.8)$ & $\geq 75$ (mean $81 \pm 4.8)$ & Mean $81 \pm 5.6$, range $65-100$ \\
\hline $\begin{array}{l}\text { Vulnerable Elders } \\
\text { Survey-13 score, } \\
\text { mean } \pm \text { SD } \text { (range) }^{10}\end{array}$ & $5.3 \pm 2.2(3-10)$ & $\begin{array}{l}4.6 \pm 2.6(1-10) \\
460(71 \%) \text { with scores } \geq 3\end{array}$ & $4.9 \pm 2.7(1-10)$ \\
\hline Conditions examined, $\mathrm{n}$ & $\begin{array}{l}15 \text { (atrial fibrillation, coronary } \\
\text { artery disease, congestive heart } \\
\text { failure, chronic renal failure, } \\
\text { dementia, diabetes mellitus, } \\
\text { depression, emphysema, fall, } \\
\text { hypertension, osteoarthritis, } \\
\text { osteoporosis, pressure ulcers, } \\
\text { stroke, urinary incontinence) }\end{array}$ & $\begin{array}{l}12 \text { (atrial fibrillation, coronary } \\
\text { artery disease, congestive heart } \\
\text { failure, dementia, diabetes } \\
\text { mellitus, depression, fall, } \\
\text { hypertension, osteoarthritis, } \\
\text { osteoporosis, stroke, urinary } \\
\text { incontinence) }\end{array}$ & $\begin{array}{l}12 \text { common (atrial fibrillation, } \\
\text { coronary artery disease, } \\
\text { congestive heart failure, } \\
\text { dementia, diabetes mellitus, } \\
\text { depression, fall, hypertension, } \\
\text { osteoarthritis, osteoporosis, } \\
\text { stroke, urinary incontinence) }\end{array}$ \\
\hline $\begin{array}{l}\text { Total comorbidity count, } \\
\text { mean } \pm \text { SD (range) }\end{array}$ & $2.3 \pm 1.5$ (range $0-7)$ & $3.5 \pm 1.8(0-10)$ & $3.1 \pm 1.8(0-10)$ \\
\hline QIs, n & $\begin{array}{l}140 \text { (covering } 22 \text { areas of care: } \\
\text { depression, diabetes mellitus, } \\
\text { dementia, falls, hearing loss, } \\
\text { congestive heart failure, } \\
\text { hypertension, coronary artery } \\
\text { disease, osteoarthritis, } \\
\text { osteoporosis, pneumonia, } \\
\text { hospitalization, pressure ulcers, } \\
\text { stroke and atrial fibrillation, } \\
\text { urinary incontinence, continuity }\end{array}$ & $\begin{array}{l}69 \text { (covering } 13 \text { areas of care: } \\
\text { dementia, depression, diabetes } \\
\text { mellitus, falls, hearing } \\
\text { impairment, hypertension, } \\
\text { malnutrition, osteoarthritis, } \\
\text { osteoporosis, pain, urinary } \\
\text { incontinence, medication use, } \\
\text { screening and prevention). } \\
\text { Participants were eligible for } \\
4 \text { to } 27 \text { Qls (mean 12). }\end{array}$ & $\begin{array}{l}61 \text { (covering } 13 \text { areas of care: } \\
\text { dementia, depression, diabetes } \\
\text { mellitus, falls, hearing } \\
\text { impairment, hypertension, } \\
\text { malnutrition, osteoarthritis, } \\
\text { osteoporosis, pain, urinary } \\
\text { incontinence, medication use, } \\
\text { screening and prevention). } \\
\text { Participants were eligible for } \\
4 \text { to } 30 \text { Qls (mean 12). }\end{array}$ \\
\hline
\end{tabular}

of care, end-of-life, malnutrition, medication management, pain, screening and prevention, and vision care). Participants were eligible for 8 to 54 Qls (mean 21).

Primary aim Observational study of feasibility of measuring ACOVE QIs

Available outcomes

Survival (days) from 0 to 3 years
Intervention at 2 of 7 sites to improve care of dementia, falls, and urinary incontinence; screening only at 5 control sites Survival (days) from 0 to 5 years Quality of life and function (Medical Health Outcomes 12item Short Form-Survey Physical Component Summary score ${ }^{20}$ ) at 1 year Participants nested within 39 different primary care physicians. Physicians cared for 1-44 participants.

Other covariables
Participants not nested within physician

Income, education
Mean $81 \pm 5.6$, range $65-100$

96

12 common (atrial fibrillation, depression, fall, hypertension, osteoarthritis, osteoporosis,

$\mathrm{SD}=$ standard deviation; $\mathrm{QI}=$ quality indicator.

score (AQS-17). Five QIs linked to better function were used to calculate the ACOVE Quality-for-Function score (AQF-5), and 16 QIs linked to improving HRQOL and symptoms to calculate the ACOVE Quality for QOL Score (AQQ-16). Participants were eligible for QOC scores if they were eligible for at least one QI in that QOC score.

\section{Outcome Measures}

Survival was calculated (in days) from enrollment with censoring at 1,143 days ( $\sim 3$ years, the duration of the shorter study, ACOVE-1). Survival data were obtained in ACOVE-1 using names, birth dates, and Social Security 
Measure Reduction:

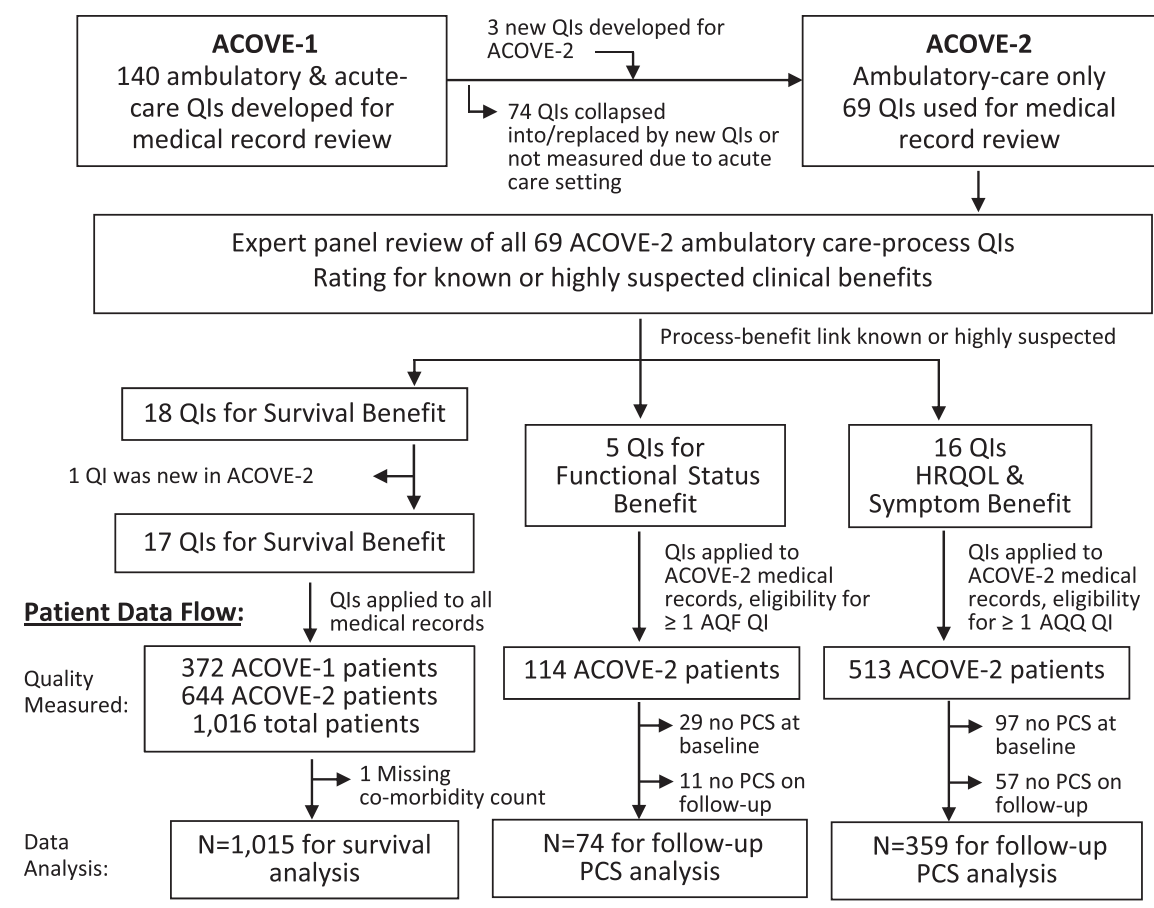

Figure 1. Measure reduction and flow of participant data. Quality indicator (QI) measure development in the Assessing Care of Vulnerable Elders Study (ACOVE-1) originally included acute and ambulatory care measures and was later reduced to ambulatory care-only measures in ACOVE-2. Only QIs measured in both studies were considered for the survival analysis on the pooled ACOVE-1 and 2 data sets, of which 17 were rated as linked (known or highly likely to be associated) with 3-year survival benefit in older adults. For five QIs rated as linked to better function and 16 QIs with health-related quality of life (HRQOL) or physical symptom benefits, composite measures of quality were tested on ACOVE-2 participants with available baseline and follow-up interviews using the Medical Outcomes Study 12-item Short-Form Survey Physical Component Summary (PCS) score.

numbers matched to the National Death Index and Social Security Master Death Files (SS-MDF), whereas ACOVE-2 used names, birth dates, and place of residence matched to death dates in the SS-MDF supplemented using obituary searches.

In ACOVE-2 only, HRQOL was collected using the Medical Outcomes Study 12-item Short Form Survey $(\mathrm{SF}-12)^{20}$ in 564 interviews during the year after enrollment (mean 10 months). Using the SF-12 responses, each participant's Physical Component Summary (PCS) score (range 0-100; 50 indicating median HRQOL related to physical function) was calculated. ${ }^{20}$ PCS score is correlated with severity of chronic disease symptoms ${ }^{20}$ and with function $^{21-23}$ and has been used as an outcome of quality of chronic disease care, ${ }^{2}$ so PCS score was used as a potential outcome of better AQQ-16 and AQF-5 scores.

\section{Covariables}

Medical records for 12 chronic conditions were reviewed in ACOVE-1 and ACOVE-2 (Table 1). Because ACOVE-2 enrolled participants based on three geriatric conditions (dementia, falls, incontinence), it had a greater mean number of conditions than ACOVE-1, which enrolled based on disabilities rather than comorbidity. Therefore, each study was divided into tertiles according to comorbidity counts: low (0-1 conditions for ACOVE-1, 0-2 for ACOVE-2), moderate (2-3 conditions for ACOVE-1, 3-4 for ACOVE-2), and severe ( $\geq 4$ conditions for ACOVE-1, $\geq 5$ for ACOVE-2). Comorbidity was tested as a main and interaction effect with quality scores.

Other covariables tested were age, sex, an indicator for ACOVE-1 versus ACOVE-2, and a modified VES-13 $3^{10}$ score (age points omitted because age was tested as a covariable).

\section{Analysis}

Data were analyzed at the level of the participant. The associations between AQS-17 and survival and between the AQF-5 and AQQ-16 and change in PCS were examined (Figure 1). $P<.05$ was considered to be statistically significant. To present the results in clinically meaningful units, QOC scores are presented according to increments of 20 absolute percentage points. If a participant was eligible for five AQS-17 QIs, then 20 percentage points is achievable by passing one additional QI.

Cox proportional hazards analysis was used to examine the association between AQS-17 and survival. Because AQS-17 violated proportional hazards assumptions, the time domain was split into two periods, fitting two separate Cox regression models. The first model tested survival from 0 to 500 days; the second from 501 to 1,143 days. The 500-day cutoff was selected by visually examining the unadjusted relationship between quality and time until death. The slope changed at approximately 500 days. 
Table 2. Quality Indicators (QIs) Rated by Expert Panel as Linked to Intended Clinical Benefits in Older Individuals in Ambulatory Care

Clinical Benefit Determined by Panel

Short Description of $\mathrm{QI}^{\mathrm{a}}$

Function

Quality of Life

Related to

Condition

\begin{tabular}{|c|c|c|c|c|}
\hline \multirow[t]{2}{*}{ All individuals } & Annual influenza vaccine & $x$ & & \\
\hline & Pneumococcal vaccine & $x$ & & \\
\hline \multirow[t]{7}{*}{ Cardiovascular disease } & Anticoagulant or antiplatelet for high-risk atrial fibrillation & $x$ & & \\
\hline & $\begin{array}{l}\text { Cholesterol intervention for low-density lipoprotein } \\
\text { cholesterol }>130 \mathrm{mg} / \mathrm{dL} \text { if CAD and failed diet intervention }\end{array}$ & $x$ & & \\
\hline & $\begin{array}{l}\text { No first- or second-generation calcium channel blockers as first- } \\
\text { line hypertension treatment }\end{array}$ & $x$ & & \\
\hline & Beta-blocker for heart failure & $\times$ & & $\times$ \\
\hline & ACE-I or ARB for heart failure & $x$ & & $x$ \\
\hline & ACE-I or ARB for hypertension and chronic renal disease & $x$ & & \\
\hline & Beta blocker after recent (2 years) myocardial infarction & $\hat{x}$ & & \\
\hline Dementia & Depression screen at initial evaluation & & & $\times$ \\
\hline \multirow[t]{4}{*}{ Depression } & New depression: document suicidality and psychosis & $x$ & & \\
\hline & Depression symptoms, screen within 2 weeks & & & $\times$ \\
\hline & Treat new depression within 2 weeks & & & $\times$ \\
\hline & Change depression treatment by 8 weeks if no response & & & $\times$ \\
\hline \multirow[t]{4}{*}{ Diabetes mellitus } & Intervention to decrease blood pressure & $x$ & & \\
\hline & Daily aspirin therapy & $\times$ & & \\
\hline & ACE-I or ARB for high cardiac risk & $x$ & & \\
\hline & Cholesterol intervention if total cholesterol $>240 \mathrm{mg} / \mathrm{dL}$ & $\times$ & & \\
\hline \multirow[t]{2}{*}{ Falls } & Exercise or assistive device for balance problem & & $x$ & $x$ \\
\hline & Exercise for strength or gait problem & & & $x$ \\
\hline Pain management & Examination for pain within 1 month & & & $\times$ \\
\hline
\end{tabular}

(17 Qls) (5 Qls)

Physical Health or Symptoms (16 QIs)

$\mathrm{CAD}=$ coronary artery disease; ACE-I = angiotensin-converting enzyme inhibitor; ARB = angiotensin receptor blocker.

${ }^{a}$ The full text of each QI is reproduced in the full table in online Appendix S2.

${ }^{\mathrm{b}}$ This QI was the 18th QI rated as having known or highly suspected survival benefit, but it was not developed until Assessing Care of Vulnerable Elders (ACOVE)-2 and therefore not tested as part of the ACOVE Quality for Survival analysis on pooled ACOVE-1 and ACOVE-2 data.

Within each of the two time periods, it was ensured that QOC no longer violated the proportional hazard assumption.

Core covariables (age, sex, modified VES-13 score, comorbidity) were first controlled for. A comorbidity-byQOC interaction term was included to test for differences in benefit between the most- and least-morbid participants. ACOVE-1 versus ACOVE- 2 was also included as a main and interaction effect with QOC in all survival analyses, which represented differences in study design and secular changes in medical care in the 3 years between the two studies (2000 and 2003, respectively). Models were also tested for consistency between men and women.
Two exploratory QI-level sensitivity analyses were performed regarding mortality to examine the individual QIs in the AQS-17 score. The first QI-level analysis was to compare mortality in those who passed with that of those who failed and review for direction of effect (Appendix S2). The binomial probability test (appropriate for small samples) was used to review for large differences in mortality between those who passed and those who failed each QI conservatively using $P<.01$ as the criterion rather than $P<.05$ because most QI-level comparisons were based on small samples with risk of Type I error. The second QI-level analysis examined whether the vaccination QIs (pneumonia and influenza) were driving the survival 
benefit because nearly all participants were eligible for them. By contrast, only half of the participants in the sample with comorbidities triggered the 15 nonvaccination QIs in the AQS-17.

To examine associations between AQF-5 and PCS and between AQQ-16 and PCS (available for ACOVE-2 only at baseline and 1 year), general linear models were used to predict change in PCS, controlling for age, sex, ACOVE-2 intervention versus control site, and comorbidity count. Comorbidity was tested for interaction effects with QOC. VES-13 was not used because of suspected colinearity with the outcome variable. Preliminary hierarchical modeling (with participants clustered within physicians and physicians within sites) were tested but did not change results from linear models. SAS (SAS Institute, Inc., Cary, NC) and SPSS (SPSS, Inc., Chicago, IL) were used for all analyses.

\section{RESULTS}

The mean age of the pooled ACOVE- 1 and ACOVE- 2 populations was 81 , and two-thirds were female (Table 1 ). Figure 1 shows how the new composite measures were applied to medical records, determining various samples from ACOVE-1 and ACOVE-2. Full data were available for 1,015 of 1,016 participants with an AQS-17 score in the pooled data sets. These participants were eligible for a total of 3,268 AQS-17 QIs (mean $3.2 \pm 1.7$, range 1-11 QIs per participant), and the mean AQS-17 score was $62 \pm 34 \%$ (interquartile range (IQR) $50-100 \%$ ). The AQQ-16 was measured in 513 of the 644 ACOVE-2 participants; baseline and follow-up PCS interviews were available for 359 of these. The AQQ-16 included one QI (pain treatment) that could be triggered multiple times per participant. These 359 participants triggered a total of 2,613AQQ-16 QIs (mean 7.3 \pm 4.9 , range 1-24 QIs per participant), and the mean AQQ-16 score was $59 \pm 31 \%$ (IQR 40-83\%). The AQF-5 was measured in 114 participants, 74 of whom had baseline and follow-up PCS interviews. These 74 participants triggered a total of 122 AQF-5 QIs (mean $1.6 \pm 0.6$, range $1-3$ ), and the mean AQF-5 score was $43 \pm 43 \%$ (IQR $0-100 \%$ ).

\section{Survival Analysis Results}

There were 68 deaths in the first 500 days: $32(8.6 \%)$ in ACOVE-1 and $36(5.6 \%)$ in ACOVE-2. There were 127 deaths between 501 days and 3 years: 54 in ACOVE-1 $(15.9 \%)$ and $73(12 \%)$ in ACOVE-2.

In the first 500 days, AQS-17 scores (Table 3, Figure 2) independently predicted survival (hazard ratio $(\mathrm{HR})=0.83$ per 20-percentage-point increment, $P=.01)$. After 500 days, there was no effect (HR $=0.98$ per 20-percentage-point increment, $P=.78)$. The VES-13 score also predicted death $(\mathrm{HR}=1.29$ per point, $P<.001)$. Having the highest level of comorbidity (but not the middle level) was associated with worse survival than having the lowest level $(\mathrm{HR}=2.19, P=.03)$. Age and sex were not related to survival. There was no difference in AQS-17 effect on survival according to comorbidity strata $\left(\mathrm{HR}_{\mathrm{QOC}} \times\right.$ moderate morbidity $=1.08, P=.161 ; \mathrm{HR}_{\mathrm{QOC}} \times$ high morbidity $=1.07$, $P=.31)$.

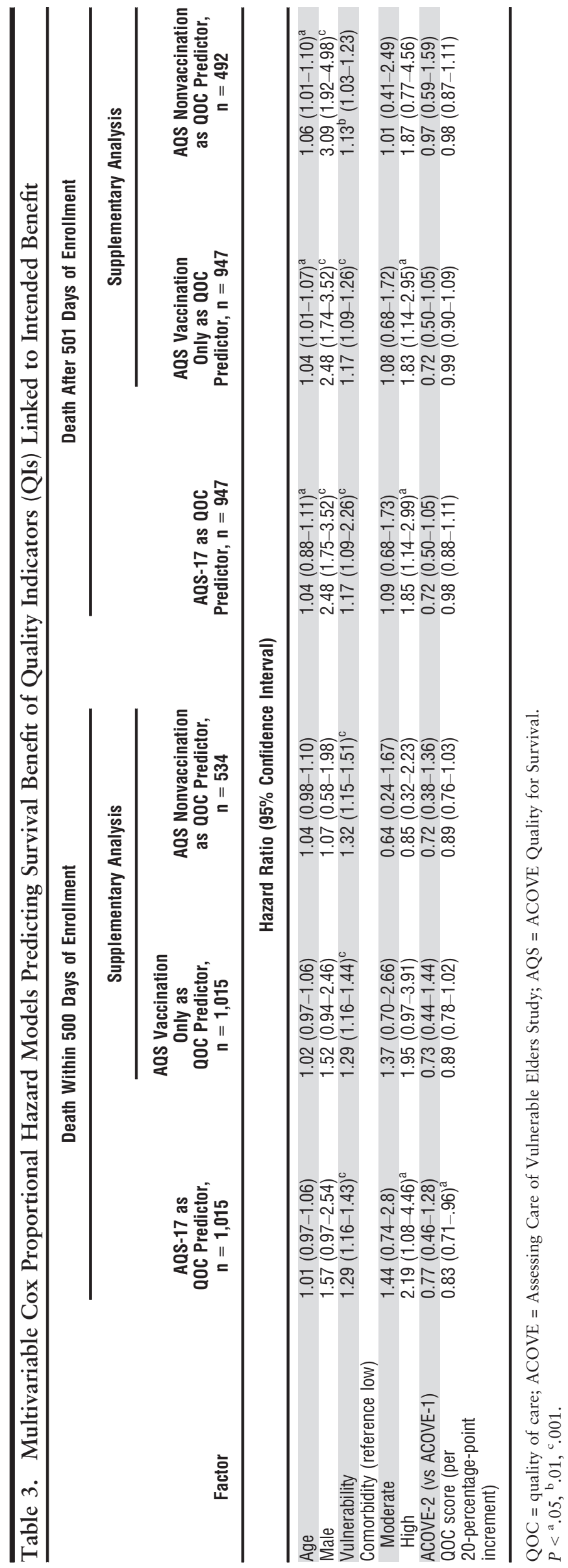




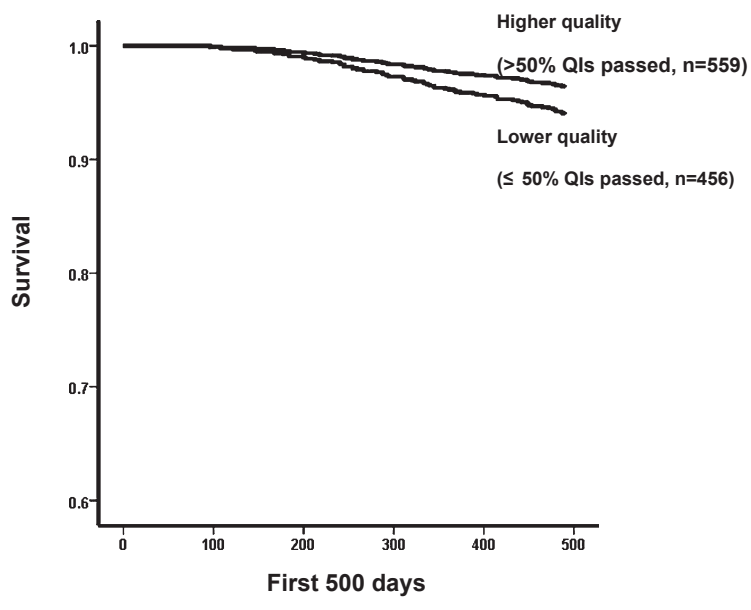

Figure 2. Survival of older participants receiving ambulatory care with higher and lower quality of care using the Assessing Care of Vulnerable Elders (ACOVE) Survival Quality score. Adjusted Kaplan-Meier curve up to 500 days, with 1,015 participants in pooled ACOVE-1 and 2 samples divided into two groups: high (score $>50 \%$, upper curve) and low (score $\leq 50 \%$, lower curve) quality. Curves adjusted for comorbidity, sex, age, ACOVE-1 versus ACOVE-2, and function-based risk (Vulnerable Elders Survey-13 score). QI = quality indicator.

\section{QI-Level Sensitivity Analyses}

Two of the 17 AQS QIs (influenza and pneumonia vaccination) were required in all participants regardless of comorbidity and were associated with more crude survival benefit than the 15 nonvaccination QIs (Appendix S2), so they were tested as two separate composite QOC scores (AQS vaccination vs AQS nonvaccination QOC score). Approximately half $(n=482)$ were eligible only for the vaccination AQS-QIs ("simple” participants). The remainder of the sample was eligible for vaccinations and at least one nonvaccination AQS-QI (534 “complex" participants). The AQS vaccination QOC score was not independently predictive in the simple, complex, or total sample. The AQS nonvaccination QOC score was not predictive in the complex sample (Table 3).

\section{Physical HRQOL Results}

The mean follow-up PCS score was $36.7 \pm 11.3$. PCS score was stable over time (mean change of +0.4 points, $P>.37$ for $t$-test of difference of two means; standard deviation of change scores $=8.7$, range 23 -point decline to 28 -point improvement). In the generalized linear models, the AQF-5 (but not the AQQ-16) predicted PCS change (more improvement or less decline). An increment of 20 percentage points in the AQF-5 score was associated with less decline in PCS score $(\beta=1.13$-point improvement, standard error (SE) $0.49, P=.02$ ), controlling for age, sex, ACOVE-2 intervention versus control group, and comorbidity. The effect size for this result was 0.27 (a small effect according to the Cohen criteria $^{24}$ ). There was no interaction between AQF-5 and intervention group. There was no effect of AQQ-16 on
PCS change score $(\beta=0.43$ points (SE 0.3) per 20-percentage-point increment in AQQ-16 score, $P>.15)$. None of the covariables (age, sex, comorbidity, intervention) predicted PCS in the AQF-5 or AQQ-16 model.

\section{DISCUSSION}

This study aimed to reduce 69 ambulatory care QIs into smaller subsets of QIs aimed at improving outcomes important to older adults. Using expert panel review, 17 primary care-processes were classified as linked to survival, and whether a composite measure of survival-oriented QIs, the AQS-17 score, would predict better survival was analyzed. A 20-percentage-point improvement on the AQS-17 was associated with $17 \%$ better 500-day survival, independent of comorbidity, sex, age, or VES-13 in older adults in primary care. This modest effect was not detected in the later observational window (500 days to 3 years), but the early benefit was consistent in those with higher versus lower levels of comorbidity burden, sex, age, and vulnerability, as well as between ACOVE-1 and ACOVE-2. Particular individual QIs did not determine the effect of the AQS-17; rather, the effect was shared between primary prevention (e.g., vaccinations) and condition-based care. A small effect of five QIs aimed at improving functional status was also found (1.13 PCS score points per 20 percentage points on the AQF-5), but not of 16 QIs aimed at improving HRQOL in a smaller sample of ACOVE-2 participants.

This observational study extends prior observational research linking better performance on care-process measures to clinical benefits, including research on vulnerable older adults ${ }^{1}$ middle-aged individuals with chronic diseases ${ }^{2}$ hospitalized older adults, ${ }^{25}$ and nursing home-eligible individuals, ${ }^{3}$ but the link between process and outcome in complex, multimorbid individuals ${ }^{26}$ with geriatric conditions (falls, urinary incontinence, dementia) has been limited to geriatric condition-specific care with condition-specific outcomes (incontinence-related quality of life $^{27}$ and falls effi$\left.\mathrm{cacy}^{28}\right)$. It is likely that it was possible to link function and survival with better performance on a composite QOC measure across conditions because care-processes were focused on according to their intended benefits.

The implication of this research is that two high-priority subsets of the ACOVE QIs can be used as a starting point for future ambulatory care initiatives to improve care and outcomes of complex older adults. The AQS-17 consisted mostly of cardiovascular care processes but also considered noncardiovascular care: preventing death in dementia (due to unsafe driving) and depression (screening for suicide). These data suggest, but do not prove, that one approach to prolonging survival might include, as a starting point, ensuring that these care process QIs are met in the care of vulnerable older adults. A 20-percentagepoint improvement in QOC, the degree of improvement that was considered to be clinically meaningful in this study, is feasible in primary care. ${ }^{9}$ Although many of the AQS-17 indicators are similar to current systems-level measures (e.g., cholesterol control), these results do not suggest that existing QIs for younger adults should be extended indefinitely in complex older adults. Rather, the less-stringent targets for vulnerable elderly adults in the 
ACOVE QIs could potentially be adopted as individuals become vulnerable (as in ACOVE-1) or develop geriatric conditions (as in ACOVE-2).

Furthermore, the results suggest that measures used in quality improvement initiatives can be customized to benefits most important to that population. For vulnerable or multimorbid older adults, personal preference may help determine whether to prioritize some care processes such as those aimed at improving function above survival. Also, because the AQS-17 can be applied to individuals with varying burdens of chronic conditions, this suggests a future approach to improving clinical outcomes in older adults with multimorbidity, who have traditionally been excluded from clinical trials. Future quality improvement aimed at longer survival or better function can target the prioritized care processes identified in the AQS-17 or AQF5 using clinical nurse specialists or care managers within primary care practices to coordinate better performance.

There are several limitations to interpreting these results. First, it is important to consider alternative explanations, for example, if poorer care was provided to individuals who were sicker (e.g., because of preference or less-aggressive care), but prior work found the opposite; sicker individuals with greater comorbidity and worse condition severity received better QOC. ${ }^{7,19,29}$ Therefore, it is unlikely that these results are due to withholding highquality care from those with multimorbidity.

Second, its measurement on a fraction of the eligible ACOVE-2 sample limited the link between a 20-percentage-point absolute improvement in AQF-5 with a 1-point improvement in PCS. A care process link with quality of life of similar magnitude has been reported in a study of middle-aged (rather than older) individuals with chronic medical conditions. ${ }^{2}$ The literature on complex interventions to improve functional status also show small or mixed benefit. ${ }^{30}$ It is imperative that better functional status outcomes measures be developed that are more sensitive to medical interventions ${ }^{31}$ and quality-of-life measures that reflect older adults' values with late-life disability. ${ }^{32}$ Therefore, if an individual highly values preserving function above survival, the focus might transition to QI sets more closely matched to his or her preferences.

Third, future survival-oriented efforts will need to be updated in response to emerging evidence of survival benefit in older adults. The newer ACOVE-3 QI set includes hundreds of new QIs not included in an expert panel review. ${ }^{33}$ New evidence that hypertension control reduces mortality ${ }^{34}$ has emerged since the expert panel was convened, but the QI concerning hypertension control could not be included in the AQS-17 because it was not measured until ACOVE-2. All of the AQS-17 QIs, such as daily aspirin for diabetes mellitus, ${ }^{35}$ need to be updated.

Fourth, this was an observational study. In contrast to trials, in which the intervention start date is known, it had to be presumed that QOC was constant over time. Participants also could have been receiving better QOC years before the observation. It is likely that the diminished survival benefit after 500 days is a limitation of the methods and should not be interpreted as a reason to stop providing high AQS-17 care after 500 days.

Fifth, the sample was predominately white, limiting generalizability of the results to minority groups. Last, it was not possible to link AQQ-16 scores with PCS scores. The PCS has limited evidence of responsiveness to changes in HRQOL in older adults. ${ }^{36}$ Better measures of HRQOL and symptoms for older adults with multimorbidities ${ }^{37}-$ and the healthcare system that can improve these outcomes $^{38}$ - are needed.

In conclusion, small sets of ambulatory care processes associated with survival even in vulnerable older adults with multimorbidity receiving ambulatory care were identified. Future effort to improve outcomes in these populations should consider improving and measuring these core subsets of care processes prioritized according to intended clinical benefit.

\section{ACKNOWLEDGMENTS}

We would like to acknowledge the technical assistance of Patty Smith, Caren Kamberg, and Chiao-Li Chan.

These results were presented at the American Geriatrics Society Annual Scientific Meeting, National Harbor, Maryland, May 14, 2011.

Conflict of Interest: The editor in chief has reviewed the conflict of interest checklist provided by the authors and has determined that the authors have no financial or any other kind of personal conflicts with this paper.

This project was funded by the Agency for Health Care Research and Quality (Min R21 HS017621). Dr. Min was also supported by the Claude Pepper Older Americans Independence Centers at the University of California at Los Angeles (NIA K12 2006-10 AG001004), the University of Michigan (NIA AG024824 2010-12), and the John A. Hartford Foundation. RAND funded the original ACOVE-1 and ACOVE-2 studies through a grant from Pfizer Corporation.

Author Contributions: Min: data acquisition, design, analysis, interpretation, drafting, revision, final approval. Reuben: interpretation, drafting, revision, final approval. Karlamangla: design, analysis, interpretation, drafting, revision, final approval. Prenovost: design, analysis, interpretation, drafting, revision, final approval. Lee: interpretation, drafting, revision, final approval. Wenger: data acquisition, interpretation, drafting, revision, final approval.

Sponsor's Role: ACOVE-2 was supported by a contract from Pfizer, Inc. to RAND. Pfizer did not play a role in the design, analysis, or preparation of this study.

\section{REFERENCES}

1. Higashi T, Shekelle PG, Adams JL et al. Quality of care is associated with survival in vulnerable older patients. Ann Intern Med 2005;143:274-281.

2. Kahn KL, Tisnado DM, Adams JL et al. Does ambulatory process of care predict health-related quality of life outcomes for patients with chronic disease? Health Serv Res 2007;42:63-83.

3. Zingmond DS, Ettner SL, Wilber $\mathrm{KH}$ et al. Association of claims-based quality of care measures with outcomes among community-dwelling vulnerable elders. Med Care 2011;49:553-559.

4. Wenger NS, Solomon DH, Roth CP et al. The quality of medical care provided to vulnerable community-dwelling older patients. Ann Intern Med 2003;139:740-747.

5. Boyd CM, Darer J, Boult C et al. Clinical practice guidelines and quality of care for older patients with multiple comorbid diseases: Implications for pay for performance. JAMA 2005;294:716-724.

6. Tinetti ME, Bogardus ST Jr, Agostini JV. Potential pitfalls of disease-specific guidelines for patients with multiple conditions. N Engl J Med 2004;351:2870-2874. 
7. Min LC, Wenger NS, Fung C et al. Multimorbidity is associated with better quality of care among vulnerable elders. Med Care 2007;45:480 488 .

8. Boyd CM, McNabney MK, Brandt $\mathrm{N}$ et al. Guiding principles for the care of older adults with multimorbidity: An approach for clinicians: American Geriatrics Society Expert Panel on the Care of Older Adults with Multimorbidity. J Am Geriatr Soc 2012;60:E1-E25.

9. Wenger NS, Roth CP, Shekelle PG et al. A practice-based intervention to improve primary care for falls, urinary incontinence, and dementia. J Am Geriatr Soc 2009;57:547-555.

10. Saliba D, Elliott M, Rubenstein LZ et al. The Vulnerable Elders Survey: A tool for identifying vulnerable older people in the community. J Am Geriatr Soc 2001;49:1691-1699.

11. Reuben DB, Roth C, Kamberg C et al. Restructuring primary care practices to manage geriatric syndromes: The ACOVE-2 intervention. J Am Geriatr Soc 2003;51:1787-1793.

12. Ganz DA, Wenger NS, Roth CP et al. The effect of a quality improvement initiative on the quality of other aspects of health care: The law of unintended consequences? Med Care 2007;45:8-18.

13. Solomon DH, Wenger NS, Saliba D et al. Appropriateness of quality indicators for older patients with advanced dementia and poor prognosis. J Am Geriatr Soc 2003;51:902-907.

14. Campbell SM, Hann M, Roland MO et al. The effect of panel membership and feedback on ratings in a two-round Delphi survey: Results of a randomized controlled trial. Med Care 1999;37:964-968.

15. Shekelle P. The appropriateness method. Med Decis Making 2004;24:228 231.

16. Shekelle PG, Chassin MR, Park RE. Assessing the predictive validity of the RAND/UCLA appropriateness method criteria for performing carotid endarterectomy. Int J Technol Assess Health Care 1998;14:707-727.

17. Brook RH, McGlynn EA, Cleary PD. Quality of health care. Part 2: Measuring quality of care. N Engl J Med 1996;335:966-970.

18. McGlynn EA, Asch SM, Adams J et al. The quality of health care delivered to adults in the United States. N Engl J Med 2003;348:2635-2645.

19. Min LC, Reuben DB, MacLean CH et al. Predictors of overall quality of care provided to vulnerable older people. J Am Geriatr Soc 2005;53:17051711.

20. Ware J Jr, Kosinski M, Keller SD. A 12-item short-form health survey: Construction of scales and preliminary tests of reliability and validity. Med Care 1996;34:220-233.

21. Johansson MM, Marcusson J, Wressle E. Cognition, daily living, and health-related quality of life in 85 -year-olds in Sweden. Neuropsychol Dev Cogn B Aging Neuropsychol Cogn 2012;19:421-432.

22. Mackenzie AE, Chang AM. Predictors of quality of life following stroke. Disabil Rehabil 2002;24:259-265.

23. Vest MT, Murphy TE, Araujo KL et al. Disability in activities of daily living, depression, and quality of life among older medical ICU survivors: A prospective cohort study. Health Qual Life Outcomes 2011;9:9.

24. Cohen J. A power primer. Psychol Bull 1992;112:155-159.

25. Arora VM, Fish M, Basu A et al. Relationship between quality of care of hospitalized vulnerable elders and postdischarge mortality. J Am Geriatr Soc 2010;58:1642-1648.

26. Min L, Wenger N, Walling AM et al. When comorbidity, aging, and complexity of primary care meet: Development and validation of the Geriatric CompleXity of Care Index. J Am Geriatr Soc 2013;61:542-550.

27. Patrick DL, Martin M, Bushnell DM. The I-QOL: A Quality-of-Life Instrument Specific to Persons with Urinary Incontinence: User's Manual and
Scoring Diskette for United States. Seattle, WA: Health Research Associates, Inc., 1999

28. Tinetti ME, Richman D, Powell L. Falls efficacy as a measure of fear of falling. J Gerontol 1990;45:P239-P243.

29. Higashi T, Wenger NS, Adams JL et al. Relationship between number of medical conditions and quality of care. N Engl J Med 2007;356:24962504.

30. Lin JS, Whitlock EP, Eckstrom E et al. Challenges in synthesizing and interpreting the evidence from a systematic review of multifactorial interventions to prevent functional decline in older adults. J Am Geriatr Soc 2012;60:2157-2166.

31. Min L, Shekelle P. Wanted: A FRAME for staging functional decline in older adults. J Am Geriatr Soc 2012;60:2167-2168.

32. King J, Yourman L, Ahalt C et al. Quality of life in late-life disability: I don't feel bitter because I am in a wheelchair. J Am Geriatr Soc 2012;60:569-576.

33. Wenger NS, Roth CP, Shekelle P. Introduction to the Assessing Care of Vulnerable Elders-3 quality indicator measurement set. J Am Geriatr Soc 2007;55(Suppl 2):S247-S252.

34. Beckett NS, Peters R, Fletcher AE et al. Treatment of hypertension in patients 80 years of age or older. N Engl J Med 2008;358:1887-1898.

35. Pignone M, Alberts MJ, Colwell JA et al. Aspirin for primary prevention of cardiovascular events in people with diabetes: A position statement of the American Diabetes Association, a scientific statement of the American Heart Association, and an expert consensus document of the American College of Cardiology Foundation. Circulation 2010;121:2694-2701.

36. Haywood KL, Garratt AM, Fitzpatrick R. Quality of life in older people: A structured review of generic self-assessed health instruments. Qual Life Res 2005;14:1651-1668.

37. Working Group on Health Outcomes for Older Persons with Multiple Chronic Conditions. Universal health outcome measures for older persons with multiple chronic conditions. J Am Geriatr Soc 2012;60:2333-2341.

38. Tinetti ME, Fried TR, Boyd CM. Designing health care for the most common chronic condition-multimorbidity. JAMA 2012;307:2493-2494.

\section{SUPPORTING INFORMATION}

Additional Supporting Information may be found in the online version of this article:

Appendix S1. Quality indicators (QIs) with full text rated by expert panel as linked with intended clinical benefits in older ambulatory care patients.

Appendix S2. Detailed 3-year mortality results for each of 17 individual quality indicators used in the AQS17, by patients who passed versus failed.

Please note: Wiley-Blackwell is not responsible for the content, accuracy, errors, or functionality of any supporting materials supplied by the authors. Any queries (other than missing material) should be directed to the corresponding author for the article. 